\title{
Controlling Structure and Porosity in Catalytic Nanoparticle Superlattices with DNA
}

\author{
Evelyn Auyeung, ${ }^{\dagger, \S}$ William Morris, ${ }^{\ddagger, \S}$ Joseph E. Mondloch, ${ }^{\ddagger}$ Joseph T. Hupp, ${ }^{\ddagger}$ Omar K. Farha, ${ }^{\ddagger}, \|$
} and Chad A. Mirkin $* \dagger, \ddagger, \S$

\author{
${ }^{\dagger}$ Department of Materials Science and Engineering, Northwestern University, 2220 Campus Drive, Evanston, Illinois 60208, United \\ States \\ ${ }^{\ddagger}$ Department of Chemistry, Northwestern University, 2145 Sheridan Road, Evanston, Illinois 60208, United States \\ ${ }^{\S}$ International Institute for Nanotechnology, Northwestern University, 2145 Sheridan Road, Evanston, Illinois 60208, United States \\ "Department of Chemistry, Faculty of Science, King Abdulaziz University, Jeddah 21589, Saudi Arabia
}

\section{Supporting Information}

\begin{abstract}
Herein, we describe a strategy for converting catalytically inactive, highly crystalline nanoparticle superlattices embedded in silica into catalytically active, porous structures through superlattice assembly and calcination. First, a body-centered cubic (bcc) superlattice is synthesized through the assembly of two sets of $5 \mathrm{~nm}$ gold nanoparticles chemically modified with DNA bearing complementary sticky end sequences. These superlattices are embedded in silica and calcined at $350{ }^{\circ} \mathrm{C}$ to provide access to the catalytic nanoparticle surface sites. The calcined superlattice maintains its bcc ordering and has a surface area of $210 \mathrm{~m}^{2} / \mathrm{g}$. The loading of catalytically active nanoparticles within the superlattice was determined by inductively coupled plasma mass spectrometry, which revealed that the calcined superlattice contained approximately $10 \%$

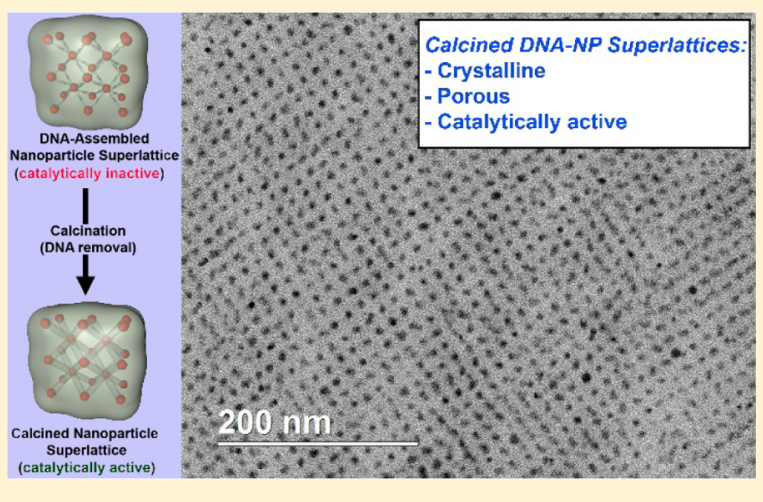
$\mathrm{Au}$ by weight. We subsequently investigate the ability of supported Au nanoparticle superlattices to catalyze alcohol oxidation. In addition to demonstrating that calcined superlattices are effective catalysts for alcohol oxidation, electron microscopy reveals preservation of the crystalline structure of the bcc superlattice following calcination and catalysis. Unlike many bulk nanoparticle catalysts, which are difficult to characterize and susceptible to aggregation, nanoparticle superlattices synthesized using DNA interactions offer an attractive bottom-up route to structurally defined heterogeneous catalysts, where one has the potential to independently control nanoparticle size, nanoparticle compositions, and interparticle spacings.
\end{abstract}

\section{INTRODUCTION}

The use of DNA to program the formation of nanoparticle superlattices has evolved into a general method that provides deliberate control over crystal symmetry, lattice parameters, composition, and, in certain cases, crystal habit. ${ }^{1-8}$ In principle, this control provides a means of realizing highly functional materials with designer optical, magnetic, electronic, and catalytic properties. In practice, it has been primarily exploited to control the optical and in particular the plasmonic properties of such structures when the particle components are made of silver or gold. ${ }^{9-11}$ From the standpoint of catalysis, the technique looks particularly attractive since it allows one to control particle size, composition, and density, while prohibiting agglomeration; however, it also poses several significant challenges that are addressed in this work. First, the DNA required for assembly necessitates that the superlattices be generated under aqueous conditions and maintained in the solution state, which limits the environments in which catalytic properties can be studied. Second, the DNA passivates the particles and adversely affects their catalytic properties. In previous work, we have shown that such superlattices can be stabilized in the solid state by encapsulating them in silica; ${ }^{12}$ however, this further limits the accessibility of the particles with respect to catalytic precursors. ${ }^{13}$ In this work, we show that following silica encapsulation, the DNA can be removed through calcination to generate porous nanoparticle superlattices that are catalytically active for alcohol oxidation. This technique allows one to prepare a particle lattice with a welldefined symmetry via the design rules ${ }^{5}$ that underlie DNA programmable assembly and rapidly convert it into a structure suitable for catalytic studies where the distribution and density of the particles are maintained and the silica has been transformed from an encasing material into a catalytic support (Scheme 1). This work is the first proof-of-concept that demonstrates the utility of DNA in nanoparticle catalysis, whereby it directs the assembly of nanoparticles into a spatially organized catalyst architectures, as well as templates the

Received: November 26, 2014

Published: January 22, 2015 
Scheme 1. Synthesis of Catalytically Active DNANanoparticle Superlattices

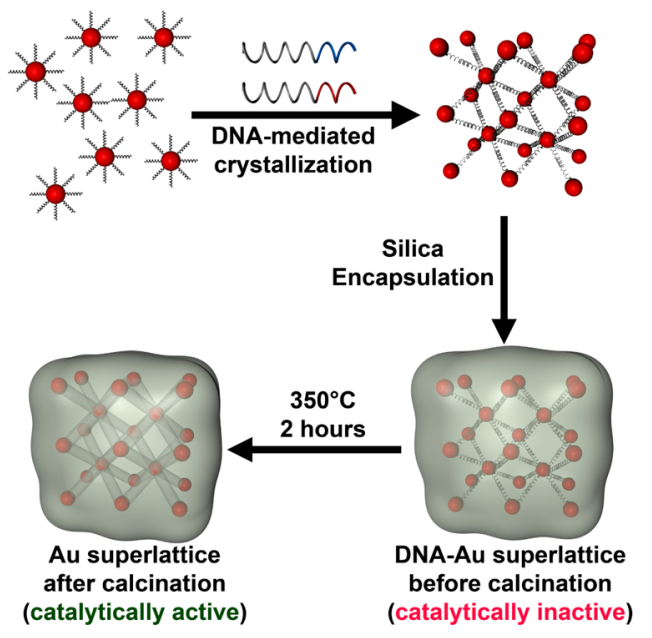

formation of porous channels that provide access to catalytically active nanoparticle surfaces.

\section{RESULTS AND DISCUSSION}

Superlattice Synthesis. First, superlattices of $5 \mathrm{~nm}$ nanoparticles with body-centered cubic (bcc) packing were synthesized using DNA assembly and silica-embedded to stabilize them in the solid state. ${ }^{14}$ Utilizing the unit cell parameters and particle size, it was possible to calculate the approximate volume occupied by $\mathrm{Au}$ nanoparticles to be approximately $1 \%$ within the superlattice. The gold content was further determined experimentally by inductively coupled plasma mass spectrometry (ICP-MS), which yielded a value of $10 \mathrm{wt} \% \mathrm{Au}$. This value is notably higher than many bulk heterogeneous catalysts prepared by metal reduction containing a random distribution of particles on a support. ${ }^{15}$ Furthermore, the bottom-up approach to superlattice synthesis allows one to incorporated highly size-monodisperse particles and increase the catalyst loading without compromising the nanoparticle dispersion within the support material, two parameters that are challenging to control in conventional supported nanoparticle catalyst systems.

Calcination. For catalytic applications, the presence of a dense environment of oligonucleotides throughout the superlattices obstructs access to the surface of the nanoparticle. To overcome this obstacle, nanoparticle superlattices were embedded in silica according to our previously developed method, ${ }^{12}$ followed by calcination at $350{ }^{\circ} \mathrm{C}$ for $2 \mathrm{~h}$ in air to remove the DNA. Thermogravimetric analysis (TGA) was conducted on a sample of silica-supported bcc superlattices, and approximately $20 \%$ weight loss was observed upon heating at $350{ }^{\circ} \mathrm{C}$ for $2 \mathrm{~h}$ (Figure 1a). ${ }^{14}$ The rapid weight decrease upon initial heating is attributed to either water absorbed during TGA sample preparation or water retained in the sample pores despite pre-measurement vacuum heating. Subsequent weight decrease is attributed to loss of DNA, which was estimated to occupy approximately $5 \%$ of the sample weight based on the expected DNA coverage per nanoparticle ${ }^{16}(\sim 25 \mathrm{dsDNA} /$ nanoparticle), as well as excess water retained within the pores. To confirm that all DNA was successfully calcined, FTIR measurements were carried out which revealed that the peak at $963 \mathrm{~cm}^{-1}$, corresponding to the skeleton motions of the $\mathrm{C}-\mathrm{C}$ and $\mathrm{C}-\mathrm{O}$ of the deoxyribose group of DNA, was completely

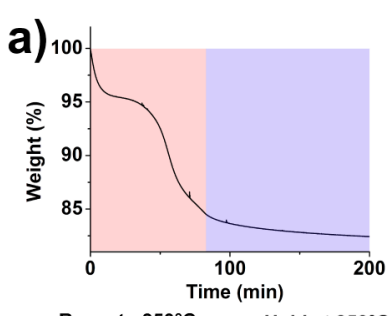

b)

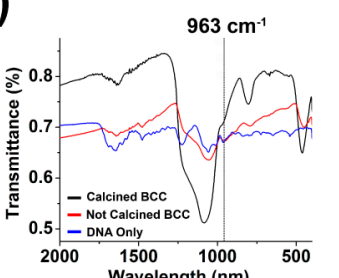

c)

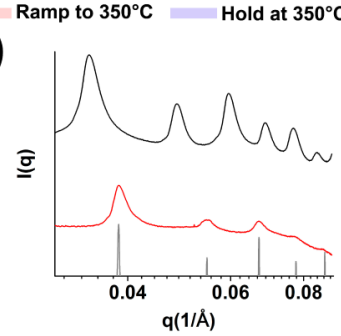

e)

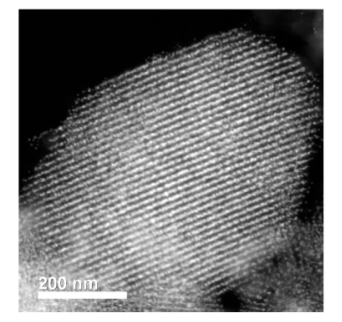

f)
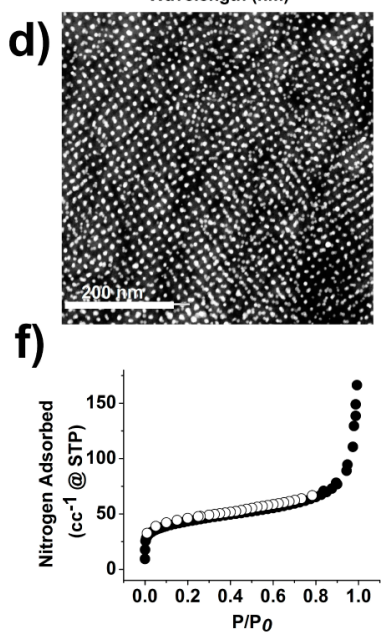

Figure 1. (a) TGA trace of silica-embedded DNA-nanoparticle superlattices. (b) FTIR spectrum of pure DNA only (blue), superlattices before calcination (red), and superlattices after calcination (black). (c) SAXS data of the as-synthesized solutionphase bcc superlattices (black) compared to the silica-embedded lattices after calcinations (red). Theoretical scattering from a perfect bcc lattice is shown in gray. (d,e) TEM images of silica-encapsulated superlattices after calcination at $350{ }^{\circ} \mathrm{C}$ for $2 \mathrm{~h}$. (f) Nitrogen adsorption (filled circles)/desorption (hollow circles) isotherms of calcined nanoparticle superlattices.

absent in the calcined sample (Figure $1 \mathrm{~b}$ ). ${ }^{17}$ Previous studies of this silica embedding process were shown to produce robust solid-state superlattices that were stable above the aggregate melting temperature (typically $40-60{ }^{\circ} \mathrm{C}$ ); however, the stability at high annealing temperatures $\left(350{ }^{\circ} \mathrm{C}\right.$ ) was not tested. Therefore, in this work, characterization of the superlattices after calcination was carried out using smallangle X-ray scattering (SAXS) and transmission electron microscopy (TEM), both of which showed that the bcc ordering of the materials is preserved with no observable aggregation or sintering after heating at $350{ }^{\circ} \mathrm{C}$ for $2 \mathrm{~h}$ (Figure $1 \mathrm{c}-\mathrm{e})$. Note that although the exact morphology of the silica was not characterized in this work, most silica types exhibit thermal stability well above the calcination temperature of 350 ${ }^{\circ} \mathrm{C},{ }^{13}$ and thus any loss of crystallinity of the superlattices due to silica decomposition was avoided. A small decrease in the lattice constant from approximately 26 to $23 \mathrm{~nm}$ is consistent with the observation in previous experiments that the lattice undergoes a slight, uniform shrinkage following silica embedding compared to the solution phase structure. The nearest neighbor spacing was calculated from the SAXS data to be approximately $20 \mathrm{~nm}$, a value that is not readily calculated for conventional supported nanoparticle catalysts due to the disordered nature of particles within the support material. Finally, to confirm access to the nanoparticle surfaces after calcination, nitrogen isotherms were collected for the calcined superlattices. Following activation at $350{ }^{\circ} \mathrm{C}$, the Brunauer- 
Emmett-Teller (BET) surface area of the calcined superlattices was calculated to be approximately $210 \mathrm{~m}^{2} / \mathrm{g}$ (Figure 1f).

Catalytic Oxidation of 4-Hydroxybenzyl Alcohol. To test whether the superlattices are catalytically active, the model reaction chosen for this work is the aerobic oxidation of 4hydroxybenzyl alcohol to the corresponding aldehyde (Figure 2), which has been extensively investigated using gold

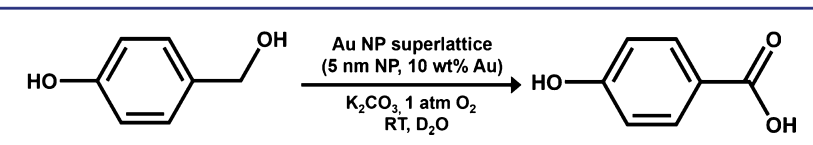

Figure 2. Reaction conditions for the superlattice-catalyzed aerobic oxidation of 4-hydroxybenzyl alcohol.

nanoparticles. ${ }^{15,18}$ The bcc superlattice catalyst $(0.215 \mathrm{nmol}$ nanoparticle) was added to a solution containing 4-hydroxybenzyl alcohol $(6.25 \mu \mathrm{mol})$ and $\mathrm{K}_{2} \mathrm{CO}_{3}(4.38 \mu \mathrm{mol})$ in $\mathrm{D}_{2} \mathrm{O}$ and the reaction was performed under 1 atm of oxygen. Yields for this reaction were determined by integration of ${ }^{1} \mathrm{H}$ peaks in ${ }^{1} \mathrm{H}$ NMR spectroscopy experiments at different time points. ${ }^{14}$ When the calcined superlattices were used as the catalyst for this reaction, $79 \%$ yield of the aldehyde was observed after $24 \mathrm{~h}$ (corresponding to a turnover number of $16000 \mathrm{~mol}$ product per $\mathrm{mol} \mathrm{Au}$ ), with $90 \%$ conversion observed after $96 \mathrm{~h}$ with no improvement in the yield after 1 week. ${ }^{14}$ This is in contrast to DNA-functionalized Au nanoparticles (unsupported) as well as the uncalcined superlattice, both samples which revealed no catalytic activity at identical particle concentrations and experimental conditions. ${ }^{14}$ Furthermore, in order to demonstrate the role of the pores defined by the calcined DNA, experiments were performed using calcined, silica-supported nanoparticles ( $0.07 \mathrm{wt} \% \mathrm{Au}, 0.02 \mathrm{nmol}$ nanoparticle) prepared through condensation of silica in the presence of pre-formed, citrate-capped $5 \mathrm{~nm}$ gold nanoparticles used to ensure uniform nanoparticle sizes throughout the support material. While the calculated BET surface area of this sample is higher than that observed for the calcined superlattice $\left(358 \mathrm{~m}^{2} / \mathrm{g}\right.$ compared to $210 \mathrm{~m}^{2} / \mathrm{g}$ due to silica comprising the majority of the sample), no conversion to the aldehyde was observed after $24 \mathrm{~h}^{14}$ This result combined with the catalytic inactivity of both the DNA functionalized particles and the non-calcined superlattice reveal that the DNA-templated pores are important for providing access to the particle surface and that DNA must be removed from surface of the gold nanoparticles to access the catalytic sites. Recycling experiments were conducted using the catalyst isolated after 24 and $72 \mathrm{~h}$ time points which resulted in $10 \%$ and $0 \%$ yields, respectively. ${ }^{14}$ This decreased catalytic activity is most likely due to contamination of the catalyst with the products of the reaction.

Evaluation of Structure following Catalytic Experiments. It was anticipated that the silica support and predefined nanoparticle spacings in the superlattices would impart stability to the system under catalytic conditions. The superlattices were collected following the reaction and characterized by SAXS and TEM, both of which showed that crystalline ordering and individual particle sizes within the superlattice were both preserved throughout catalysis (Figure 3 ). Thus, in addition to allowing for precise structural characterization, these crystalline superlattices possess the advantage of stability against catalyst agglomeration or sintering due to the well-defined positions of the nanoparticles in the pre-formed lattice.

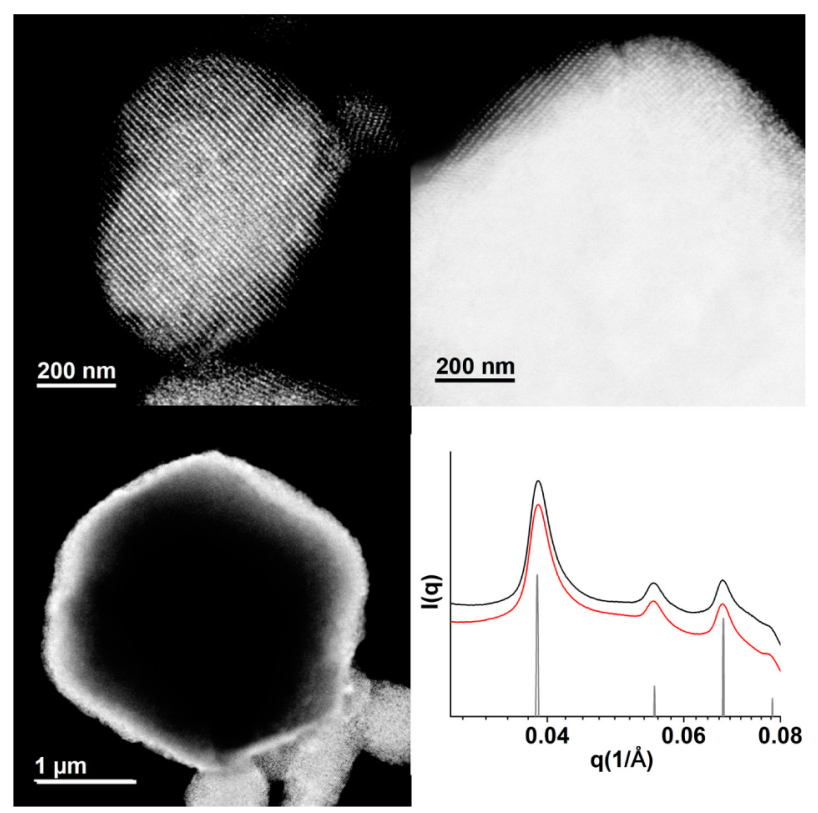

Figure 3. TEM images and SAXS data of the superlattices after catalysis (24-h time point): black trace, before catalysis; red trace, after catalysis; gray trace, scattering from a perfect bcc lattice.

\section{CONCLUSIONS}

In summary, we have synthesized the first catalytically active nanoparticle superlattices assembled using DNA interactions. A simple three-step process was used to synthesize the catalytically active superlattices, which maintain their crystalline order throughout calcination and catalysis. Importantly, we envision many catalyst systems will be synthesized and studied using this strategy due to the ability of DNA-programmable assembly to allow for precise and independent control over parameters relevant in catalysis including nanoparticle composition, nanoparticle size, and interparticle spacing. Furthermore, this strategy of creating heterogeneous catalysts with controlled structures and high particle loading is a significant contribution in the field of nanoparticle catalysis, in which the majority of catalysts suffer from structural inhomogeneity and are thus difficult if not impossible to structurally characterize. ${ }^{19}$ In addition to the aerobic alcohol oxidation demonstrated in this work, these nanoparticle superlattices may be useful catalysts for a number of common gold-catalyzed reactions ${ }^{15,20}$ including alcohol oxidation, ${ }^{18} \mathrm{CO}$ oxidation, ${ }^{21}$ and plasmon-mediated reduction reactions. ${ }^{22}$ The work described herein will also serve to inform many research directions in the development of these materials as highly efficient catalysts for a variety of important transformations, where variables that affect catalytic activity such as particle density and particle composition (e.g., platinum nanoparticles ${ }^{23}$ or a binary lattice of gold and platinum for tandem catalysis ${ }^{24}$ ) can all be readily modulated through the use of the DNA design rules.

\section{EXPERIMENTAL SECTION}

Materials and General Procedures. Unless otherwise stated, all reagents were purchased from commercial sources and used without further purification. Gold nanoparticles of $5 \mathrm{~nm}$ diameter were purchased from Ted Pella. 4-Hydroxybenzyl alcohol, potassium carbonate $\left(\mathrm{K}_{2} \mathrm{CO}_{3}\right)$, dithiothreitol (DTT), Tween-20, sodium chloride, and triethoxysilane (TES) were purchased from SigmaAldrich. $\mathrm{N}$-Trimethoxysilylpropyl- $\mathrm{N}, \mathrm{N}, \mathrm{N}$-trimethylammonium chloride 
was purchased from Gelest, Inc. Deuterium oxide $\left(\mathrm{D}_{2} \mathrm{O}\right)$ was purchased from Cambridge Isotope Laboratories and used as received.

All oligonucleotides used in this work were synthesized on a MM12 synthesizer (Bioautomation) fitted with $5-\mu$ mol-scale columns and purified according to the procedures described previously. ${ }^{1,3,5}$ Sequences can be found in the Supporting Information. SAXS data were collected at the DuPont-Northwestern-Dow Collaborative Access Team (DND-CAT) beamline at Argonne National Laboratory's Advanced Photon Source with $10 \mathrm{keV}$ (wavelength $\lambda=1.24 \AA$ ) collimated X-rays calibrated with a silver behenate standard. TEM images were collected on a Hitachi HD-2300 scanning transmission electron microscope. Gas adsorption isotherms were measured volumetrically on an Autosorb-1 analyzer (Micromeritics). FTIR experiments were performed on a Bruker Tensor 37 spectrometer. ${ }^{1} \mathrm{H}$ NMR spectra were collected on a Bruker Avance $400 \mathrm{MHz}$ spectrometer.

Nanoparticle Assembly and Crystallization. For the experiments detailed in this work, nanoparticle functionalization with DNA was carried out at an approximately 10 -fold larger scale than conventional DNA assembly work ${ }^{1,3,5}$ in order to produce amounts sufficient for catalysis and related experiments. Briefly, approximately $500 \mathrm{nmol}$ of the thiolated sequences (sequences $\mathbf{1}$ and $\mathbf{2}$ in the Supporting Information) was reduced with $100 \mathrm{mM} \mathrm{DTT}$ at $\mathrm{pH} 8$ and purified through a Nap-25 Sephadex size-exclusion column. The thiolated DNA was added to a solution of citrate-capped gold nanoparticles stabilized with $0.01 \%$ volume of the surfactant Tween 20 at a concentration of approximately $5 \mathrm{nmol}$ of DNA per $\mathrm{mL}$ of particles. Following DNA addition, the particles were placed on a shaker at room temperature for approximately $30 \mathrm{~min}$ before subsequent salt addition. In order to maximize DNA loading on the particle surface, $\mathrm{NaCl}$ was slowly added over the course of several hours until the final salt concentration reached $0.5 \mathrm{M} \mathrm{NaCl}$. After allowing the particle solution to shake overnight at room temperature, the particles were purified by five rounds of centrifugation $(10 \mathrm{~min}$, $4000 \mathrm{rpm}$ ) using $10 \mathrm{~K}$ molecular weight cutoff spin filters (Millipore) to remove the salt and any unbound DNA. After the final centrifugation, the supernatant was removed and salt was added to bring the final salt concentration of the solution to $0.5 \mathrm{M} \mathrm{NaCl}$. DNA and DNA-functionalized nanoparticle concentrations were calculating using Beer's law with the absorbance (at $260 \mathrm{~nm}$ for the DNA and at $520 \mathrm{~nm}$ for the Au nanoparticles) measured on a Cary 5000 (Agilent) $\mathrm{UV} /$ vis spectrophotometer. To synthesize a bcc superlattice, two solutions of particles functionalized with strands $\mathbf{1}$ and $\mathbf{2}$ were added in a 1:1 ratio, followed by the addition of linker strands 3 and 4 that initiate rapid particle aggregation (see Supporting Information for sequences). The aggregates (disordered at this stage) were subsequently transferred to PCR tube strips and slow-cooled in a Veriti temperature cycler (Life Technologies) from 60 to $25{ }^{\circ} \mathrm{C}$ at a rate of $0.1{ }^{\circ} \mathrm{C} / 10 \mathrm{~min}$ to produce crystallized nanoparticle superlattices with bcc packing.

Silica Embedding. Superlattices were transferred to the solid state using the modified silica embedding method as previously described. $^{3,12}$ As-synthesized superlattices were divided into $1.5 \mathrm{~mL}$ Eppendorf tubes containing approximately $500 \mu \mathrm{L}$ sample volume, followed by the addition of $10 \mu \mathrm{L}$ of TMSPA and $20 \mu \mathrm{L}$ of TES into each tube. The reaction was allowed to shake for 4 days on a thermomixer (Eppendorf), followed by purification by centrifugation to remove excess silica and precursor molecules. The purified superlattices were dried in a CentriVap vacuum concentrator (Labconco) to yield a dark purple solid.

Preparation of Supported, Non-assembled Gold Nanoparticles. To form the non-assembled, supported nanoparticle catalyst, pre-formed $5 \mathrm{~nm}$ diameter Au nanoparticles (not functionalized with DNA) were added to a silica growth solution to ensure that particle sizes would be uniform across all catalysis experiments. Next, $100 \mathrm{~mL}$ of citrate-capped Au nanoparticles $\left(5.0 \times 10^{13}\right.$ particles $/ \mathrm{mL}$, as reported by the manufacturer) was added to a round-bottom flask equipped with a stir bar. This was followed by the addition of $1 \mathrm{~mL}$ TES (same silica precursor as was used in the silica encapsulation process described above). The reaction was allowed to stir overnight and subsequently purified by at least 10 rounds of centrifugation and resuspension in Nanopure water to remove all unbound nanoparticles. The pellet was collected and dried in a CentriVap vacuum concentrator (Labconco) to yield a pink solid. Samples were calcined at $350{ }^{\circ} \mathrm{C}$ for $2 \mathrm{~h}$ prior to catalysis experiments. Gold content was measured by inductively coupled plasma mass spectrometry. See Supporting Information for TEM images of the silica-supported, nonassembled Au nanoparticles.

Calcination of Superlattice Assemblies. Dried, silica-encapsulated superlattices were transferred to $3 \mathrm{~mm}$ quartz capillaries (Charles Supper) and placed inside a Lindberg Blue $M$ tube furnace (Thermo Scientific). Samples were heated in air at $350{ }^{\circ} \mathrm{C}$ for $2 \mathrm{~h}$ (not including the $1 \mathrm{~h}$ ramp time to $350{ }^{\circ} \mathrm{C}$ ).

Thermogravimetric Analysis (TGA). Samples were dried under reduced pressure at $60{ }^{\circ} \mathrm{C}$ for $6 \mathrm{~h}$ in preparation for TGA measurements. TGA was performed on a Discovery TGA (TA Instruments). The temperature was ramped to $350{ }^{\circ} \mathrm{C}$ at a rate of 4 ${ }^{\circ} \mathrm{C} / \mathrm{min}$ and held at $350{ }^{\circ} \mathrm{C}$ for $10 \mathrm{~h}$.

Fourier Transform Infrared Spectroscopy (FTIR). Superlattices dissolved in toluene were drop cast on a $\mathrm{KBr}$ Real Crystal IR sample card (International Crystal Laboratories). Spectra (averaged over 16 scans) were collected in transmission mode and corrected for $\mathrm{H}_{2} \mathrm{O}$ and $\mathrm{CO}_{2}$ in the OPUS software.

$\mathbf{N}_{\mathbf{2}}$ Isotherm Measurements. A liquid nitrogen sample bath (77 $\mathrm{K})$ was used, and the $\mathrm{N}_{2}$ gas used was UHP grade. For measurement of the apparent surface areas $\left(S_{\mathrm{BET}}\right)$, the BET method was applied using the adsorption branches of the $\mathrm{N}_{2}$ isotherms assuming a $\mathrm{N}_{2}$ cross-sectional area of $16.2 \AA^{2} /$ molecule.

Small-Angle X-ray Scattering (SAXS). Exposure times of 0.1 and $1 \mathrm{~s}$ were used for solution-phase lattices and silica-embedded samples, respectively. X-ray scattering data were collected on a CCD area detector, and one-dimensional patterns were obtained from radial averaging of the two-dimensional data to generate plots of scattering intensity $I(q)$ as a function of the scattering vector $q(q=4 \pi \sin \theta / \lambda$, where $\theta$ and $\lambda$ are the scattering angle and wavelength of the $\mathrm{X}$-rays used, respectively). Theoretical SAXS patterns were generated using PowderCell (available free of charge from the Federal Institute for Materials Research and Testing at http://www.ccp14.ac.uk/ccp/webmirrors/powdcell/a_v/v_1/powder/e_cell.html), a software package that simulates scattering patterns for atomic lattices but which provides a good approximation of SAXS patterns obtained for the nanoparticle lattices studied in this work. The nanoparticle nearest-neighbor distance $(d$, in $\mathrm{nm})$ was calculated using the position of the first scattering peak $q_{0}$ in the SAXS pattern:

$$
d=\frac{1}{10} \frac{\sqrt{6 \pi}}{q_{0}}
$$

Lattice parameters were calculated from the nearest neighbor distance using geometric considerations for a bcc unit cell.

Inductively Coupled Plasma Mass Spectrometry (ICP-MS). Gold content was measured by ICP-MS measurements carried out in Thermo X series II instrument with an automated sample changer. Dried superlattices were dispersed homogeneously in $2 \mathrm{~mL}$ of $\mathrm{D}_{2} \mathrm{O}$. From this stock solution, $5 \mu \mathrm{L}$ was added to $95 \mu \mathrm{L}$ of $\mathrm{D}_{2} \mathrm{O}$, and $5 \mu \mathrm{L}$ of this solution was digested in $995 \mu \mathrm{L}$ of aqua regia. The solution containing superlattices in aqua regia was left at $60^{\circ} \mathrm{C}$ overnight to ensure full digestion of the nanoparticles. Samples were prepared with a multi-element standard and compared to a standard curve generated using a gold standard solution.

Catalysis Experiments. In a typical catalysis experiment, the catalyst (0.215 nmol Au nanoparticles), 4-hydroxybenzyl alcohol (6.25 $\mu \mathrm{mol})$, and $\mathrm{K}_{2} \mathrm{CO}_{3}(4.38 \mu \mathrm{mol})$ were combined in $2.5 \mathrm{~mL}$ of $\mathrm{D}_{2} \mathrm{O}$ and added to a $20 \mathrm{~mL}$ round-bottom flask containing a magnetic stir bar. In the case of the supported, non-assembled particles that contained low nanoparticle loading compared to the superlattice sample $(0.07 \mathrm{wt}$ $\%$ compared to $10 \mathrm{wt} \%)$, side-by-side experiments using the calcined superlattices containing an equivalent molar amount of nanoparticles $(0.02 \mathrm{nmol})$ were performed to compare the catalytic activity. The flask containing the starting materials was fitted with a rubber septum 
and purged with a balloon filled with oxygen $\left(\mathrm{O}_{2}\right)$ gas. The balloon was refilled with $\mathrm{O}_{2}$ gas and attached through the septum for the duration of the reaction. The reaction proceeded at room temperature for all specified time points. Separate flasks were used for each time point, after which approximately $600 \mu \mathrm{L}$ of the reaction solution was transferred to NMR tubes for characterization by ${ }^{1} \mathrm{H}$ NMR spectroscopy.

For the two recycling experiments, the reaction was left for 24 and $72 \mathrm{~h}$, respectively, followed by isolation of the catalyst by three rounds of centrifugation and resuspension with $\mathrm{D}_{2} \mathrm{O}$.

\section{ASSOCIATED CONTENT}

\section{S Supporting Information}

Oligonucleotide sequences, TEM images, and ${ }^{1} \mathrm{H}$ NMR spectra. This material is available free of charge via the Internet at http://pubs.acs.org.

\section{AUTHOR INFORMATION}

\section{Corresponding Author}

*chadnano@northwestern.edu

\section{Notes}

The authors declare no competing financial interest.

\section{ACKNOWLEDGMENTS}

C.A.M. acknowledges support from the Office of the Assistant Secretary of Defense for Research and Engineering, DoD/ NSSEFF Program, under Award No. N00014-15-1-0043 and the AFOSR under award numbers FA9550-14-1-0274 and FA9550-11-1-0275. O.K.F. gratefully acknowledges funding from the Army Research Office (W911NF-13-1-0229). TEM images were collected at the Electron Probe Instrumentation Center of the Northwestern University Atomic and Nanoscale Characterization Experimental Center, supported by NSFNSEC, NSF-MRSEC, Keck Foundation, the State of Illinois, and Northwestern University. SAXS data were collected at the Dupont-Northwestern-Dow Collaborative Access Team (DND-CAT) Beamline 5-IDD at Argonne National Laboratory. Use of the DNA-CAT beamline was supported by the U.S. DOE, Office of Science, Office of Basic Energy Sciences, under contract DE-AC02-06CH11357. We thank Nikunjkumar Savalia for his assistance with DNA synthesis and purification.

\section{REFERENCES}

(1) Park, S. Y.; Lytton-Jean, A. K. R.; Lee, B.; Weigand, S.; Schatz, G. C.; Mirkin, C. A. Nature 2008, 451, 553.

(2) Nykypanchuk, D.; Maye, M. M.; van der Lelie, D.; Gang, O. Nature 2008, 451, 549.

(3) Auyeung, E.; Li, T. I. N. G.; Senesi, A. J.; Schmucker, A. L.; Pals, B. C.; de la Cruz, M. O.; Mirkin, C. A. Nature 2014, 505, 73.

(4) Zhang, C.; Macfarlane, R. J.; Young, K. L.; Choi, C. H. J.; Hao, L. L.; Auyeung, E.; Liu, G. L.; Zhou, X. Z.; Mirkin, C. A. Nat. Mater. 2013, 12, 741 .

(5) Macfarlane, R. J.; Lee, B.; Jones, M. R.; Harris, N.; Schatz, G. C.; Mirkin, C. A. Science 2011, 334, 204.

(6) Macfarlane, R. J.; Jones, M. R.; Senesi, A. J.; Young, K. L.; Lee, B.; Wu, J. S.; Mirkin, C. A. Angew. Chem., Int. Ed. 2010, 49, 4589.

(7) Jones, M. R.; Macfarlane, R. J.; Lee, B.; Zhang, J. A.; Young, K. L.; Senesi, A. J.; Mirkin, C. A. Nat. Mater. 2010, 9, 913.

(8) Macfarlane, R. J.; O’Brien, M. N.; Petrosko, S. H.; Mirkin, C. A. Angew. Chem., Int. Ed. 2013, 52, 5688.

(9) Young, K. L.; Ross, M. B.; Blaber, M. G.; Rycenga, M.; Jones, M. R.; Zhang, C.; Senesi, A. J.; Lee, B.; Schatz, G. C.; Mirkin, C. A. Adv. Mater. 2014, 26, 653.

(10) Ross, M. B.; Blaber, M. G.; Schatz, G. C. Nat. Commun. 2014, 5, 4090
(11) Tan, S. J.; Campolongo, M. J.; Luo, D.; Cheng, W. L. Nat. Nanotechnol. 2011, 6, 268.

(12) Auyeung, E.; Macfarlane, R. J.; Choi, C. H. J.; Cutler, J. I.; Mirkin, C. A. Adv. Mater. 2012, 24, 5181.

(13) Joo, S. H.; Park, J. Y.; Tsung, C. K.; Yamada, Y.; Yang, P. D.; Somorjai, G. A. Nat. Mater. 2009, 8, 126.

(14) See Supporting Information for oligonucleotide sequences, ${ }^{1} \mathrm{H}$ NMR spectra, and TEM images.

(15) Hashmi, A. S. K.; Hutchings, G. J. Angew. Chem., Int. Ed. 2006, 45, 7896.

(16) Hill, H. D.; Millstone, J. E.; Banholzer, M. J.; Mirkin, C. A. ACS Nano 2009, 3, 418.

(17) Mello, M. L. S.; Vidal, B. C. Plos One 2012, 7.

(18) Tsunoyama, H.; Sakurai, H.; Negishi, Y.; Tsukuda, T. J. Am. Chem. Soc. 2005, 127, 9374.

(19) Bell, A. T. Science 2003, 299, 1688.

(20) Mikami, Y.; Dhakshinamoorthy, A.; Alvaro, M.; Garcia, H. Catal. Sci. Technol. 2013, 3, 58.

(21) Zanella, R.; Giorgio, S.; Shin, C. H.; Henry, C. R.; Louis, C. J. Catal. 2004, 222, 357.

(22) Zhu, H. Y.; Ke, X. B.; Yang, X. Z.; Sarina, S.; Liu, H. W. Angew. Chem., Int. Ed. 2010, 49, 9657.

(23) Chen, J. Y.; Lim, B.; Lee, E. P.; Xia, Y. N. Nano Today 2009, 4 81.

(24) Yamada, Y.; Tsung, C. K.; Huang, W.; Huo, Z. Y.; Habas, S. E.; Soejima, T.; Aliaga, C. E.; Somorjai, G. A.; Yang, P. D. Nat. Chem. 2011, 3, 372. 